\title{
Research on the Carbonation Model of Stress Concrete Components
}

\author{
Ma Rui ${ }^{1, a}$, Zhang Jin-Quan ${ }^{1}$,Diao Bo ${ }^{2}$ \\ ${ }^{1}$ Bridge Technology Research Center Research Institute of Highway Ministry of Transport, Beijing, \\ 100088, China \\ ${ }^{2}$ BeihangUniversity, Beijing, 100191, China \\ a124664250@qq.com
}

Keywords: Relative carbonation depth; Standard carbonation depth; Stress concrete components;

\begin{abstract}
Research results on the stress of concrete member carbonization are rich, as many as dozens of mathematical prediction models. But the Achievements on the Stress concrete components are less. In this paper, based on the research of the existing test date, a new method of calculating the depth of stress concrete components was proposed, which can not only make full use of the existing research results on the concrete components without stress, but also can use experimental data form different references. And the prediction model of the carbonation depth of the stress concrete components was put forward and verified
\end{abstract}

\section{Introduction}

In atmospheric environment, the concrete structures in service always carbonized. Many researches were done to study the carbonization of stressless concrete components, and many mathematical prediction models have been proposed. But the Achievements on the Stress concrete components are very few.

References [1]-[6] did experiments on the stress concrete components. Restricted by experimental condition, Test samples are few in number, generally 3 to 6 samples. So it was difficult to obtain statistical law.

In this paper, on the basis of the analysis of the existing test data, a new method to calculate the stress concrete components' carbonation depth was put forward, and also the calculation model was obtained. By using this method can extend the sample space, get the corresponding statistical law.

\section{Calculation model of the stress concrete carbonation depth}

The carbonization process of concrete material is complex, affected by many factors. References [8]-[9] did many comparison and analysis on the existing carbonation models, but these models were not included the influence of working conditions. The concrete structure members are under loading, on the basis of an analysis of material, the most commonly exist stress is tensile stress or compressive stress. Due to micro cracks exist in concrete material, the compressive stress will make part of the micro crack closure or propagation velocity is reduced, while the tensile stress will make the micro crack increase, so the existing stress can affect the carbonization rate.

Consider the influence of stress on the carbonization speed, absorbing the existing research results, combining with the characteristics of carbonization. In this paper, the carbonation depth of stress concrete components is divided into two parts: one part is the carbonation depth of the components without stress, defined as Standard Carbonation Depth; another part is the carbonation depth of the stress components increase or decrease relative to Standard Carbonation Depth, defined as Relative Carbonation Depth. Therefore, the carbonation depth of the stress concrete components can be written as follows:

$$
x_{c}=x_{o}+x_{\sigma}
$$

In the type: $x_{c}$ is the carbonation depth of the stress concrete components $(\mathrm{mm}) ; x_{0}$ is the Standard Carbonation Depth(mm); $x_{\sigma}$ is the Relative Carbonation Depth(mm), reflects the stress increase 
or decrease the carbonation depth.

All the references on the carbonation believed that the $\mathrm{CO} 2$ propagation speed is proportional to the square root of time, according to this law, relative carbonation depth formula is given as follows:

$$
x_{\sigma}=A_{0} \chi_{\sigma} \sqrt{t}
$$

In the type: A0 is a constant, which will be determined using experimental methods; $\chi_{\sigma}$ is the item related to stress. In order to use the results from different experiments, $\chi_{\sigma}$ is made as a dimensionless value, takes $\chi_{\sigma}=\frac{\sigma}{f_{t k}}$ when there is tensile stress, takes $\chi_{\sigma}=\frac{\sigma}{f_{c k}}$ when there is compressive stress; tis for accelerated carbonation test time(d).

\section{Mathematical model validation}

Reference [6] did experimental studies on the stress concrete components, using the existing test dates to verify formulas in this paper.

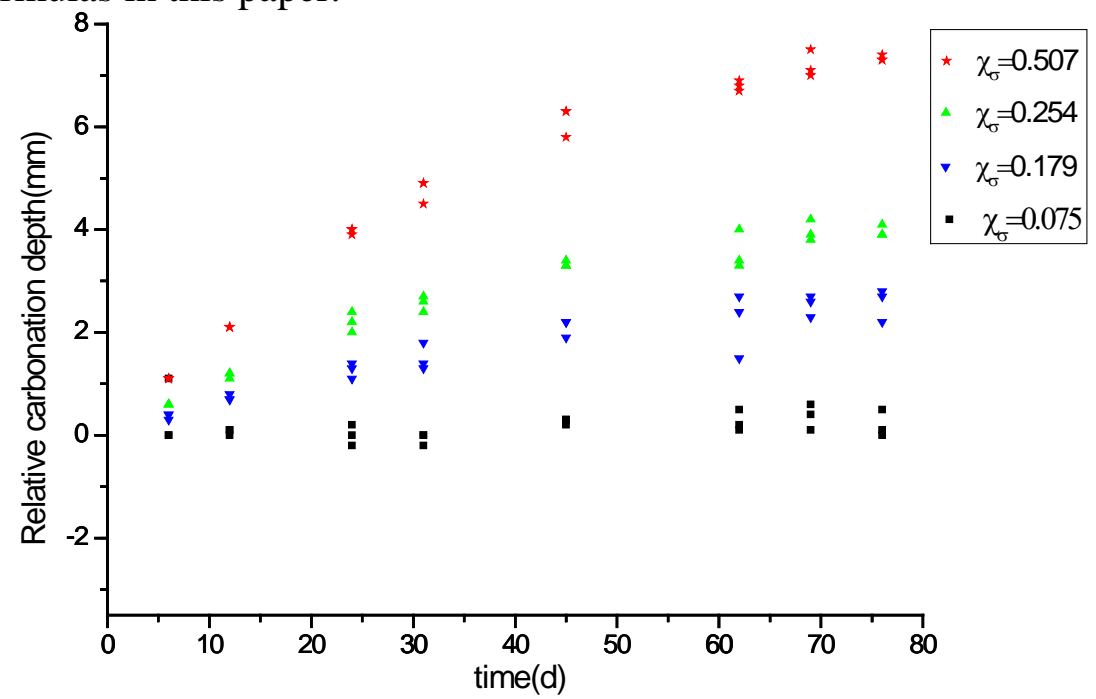

(1) the tensile stress state

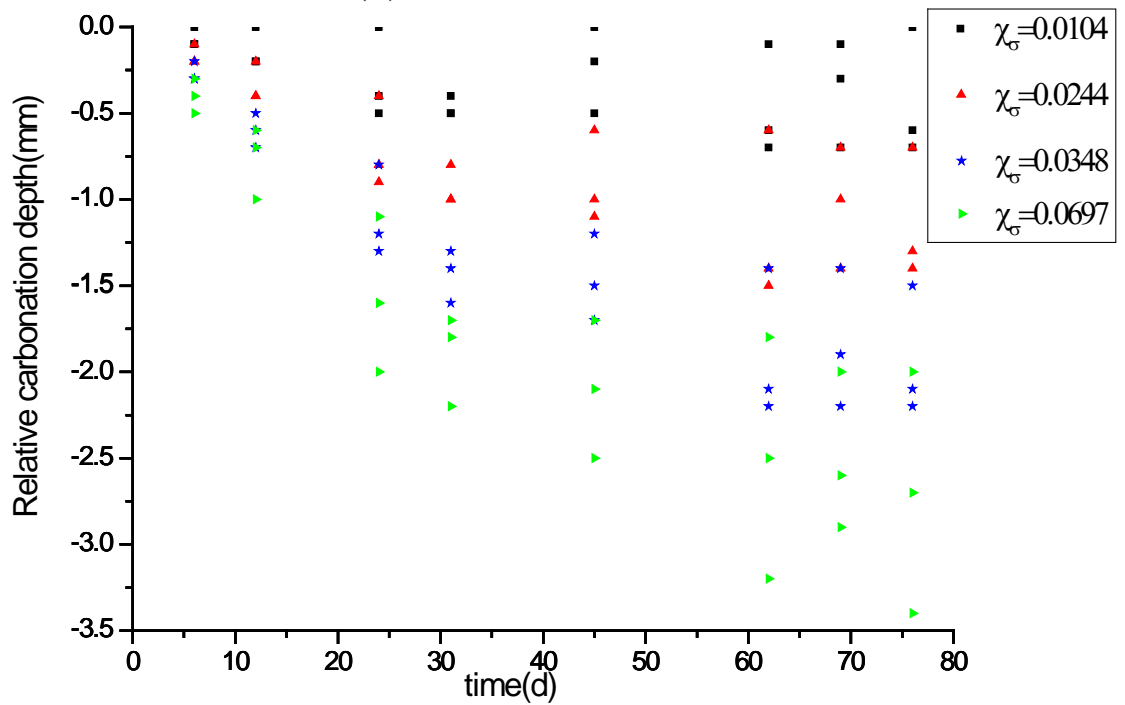

(2)the Compressive stress state

Fig 1. The influence of Concrete composition on carbonation depth

Note: Each data atthe same time represents different water cement ratio $(0.4,0.48,0.55)$

According to the Fig 1, in tensile stress state, the Relative Carbonation Depth data is 
concentrated, while in compressive stress state, the Relative Carbonation Depth data shows a trend of diffusion as the time growth.On the whole, the Relative Carbonation Depths under the three different conditions are consistent. The equation (1) introduces Relative Carbonation Depth to consider the impact of stress on the depth of carbonation separately is reasonable.

Fig2 and Fig3 show the data from references [1]-[7]. It can be seen that using the method in this paper, all the date form different experiments can be used to analysis the Relative Carbonation Depth together.

According to the Fig2, the Relative Carbonation Depth increases while the tensile stress growth. And also it increases while the time growth.

According to the Fig3, the absolute values of Relative Carbonation Depth increases while compressive stress growth, and also it increases while the time growth.

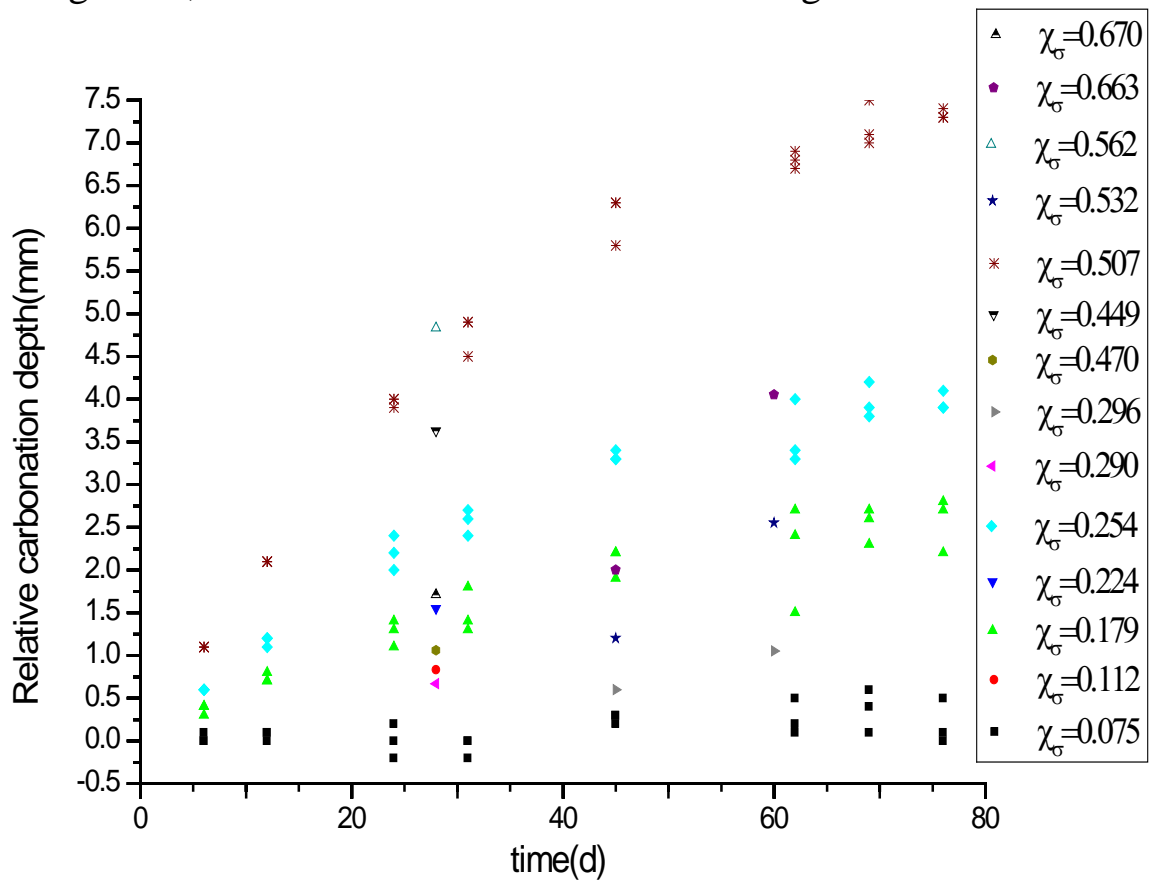

Fig 2. The Relative carbonation depth with different tensile stress

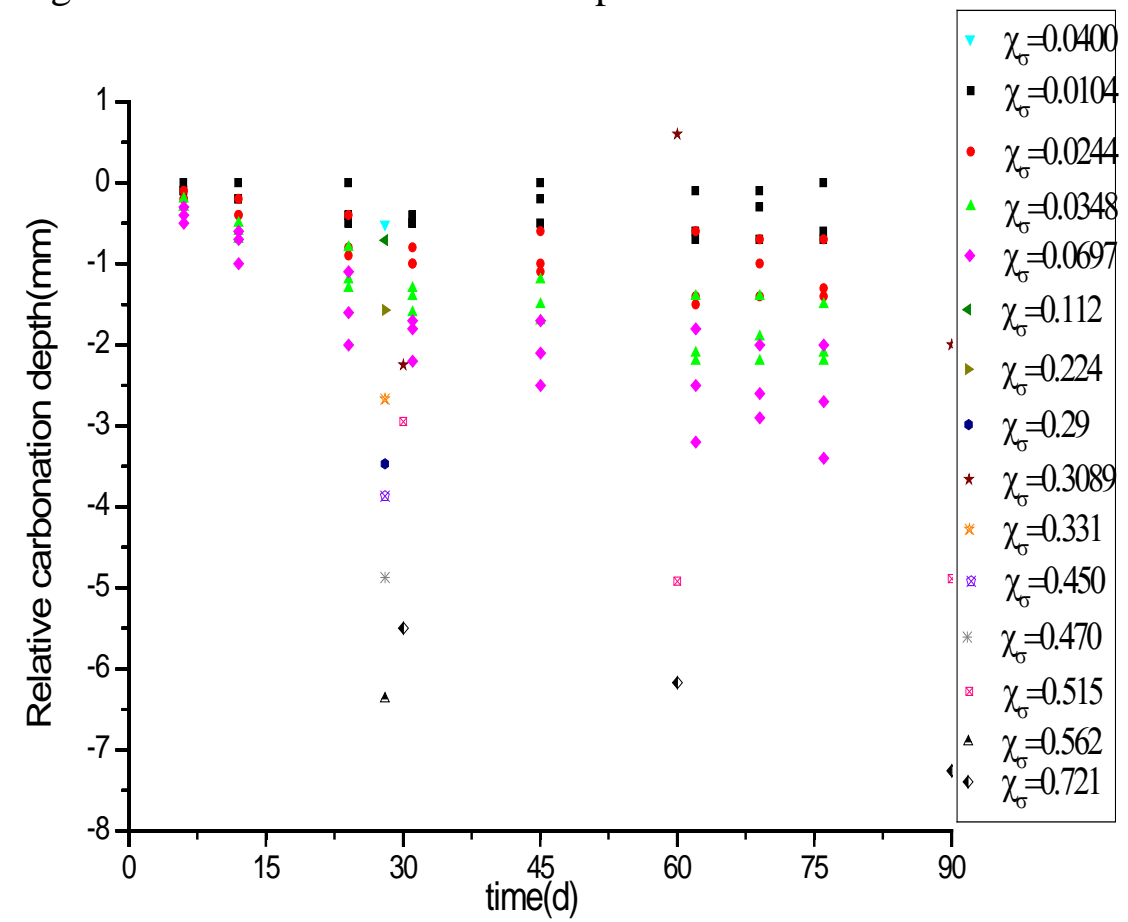

Fig 3. The Relative carbonation depth with different compressive stress

In summary, the tensile stress can increase the carbonization speed, while the compressive stress effectively reducing the seed of carbide. The above data came from different references, which did 
the experiments under different conditions, including different materials, water cement ration and so on. So the influence of such factors, some data is fluctuated.

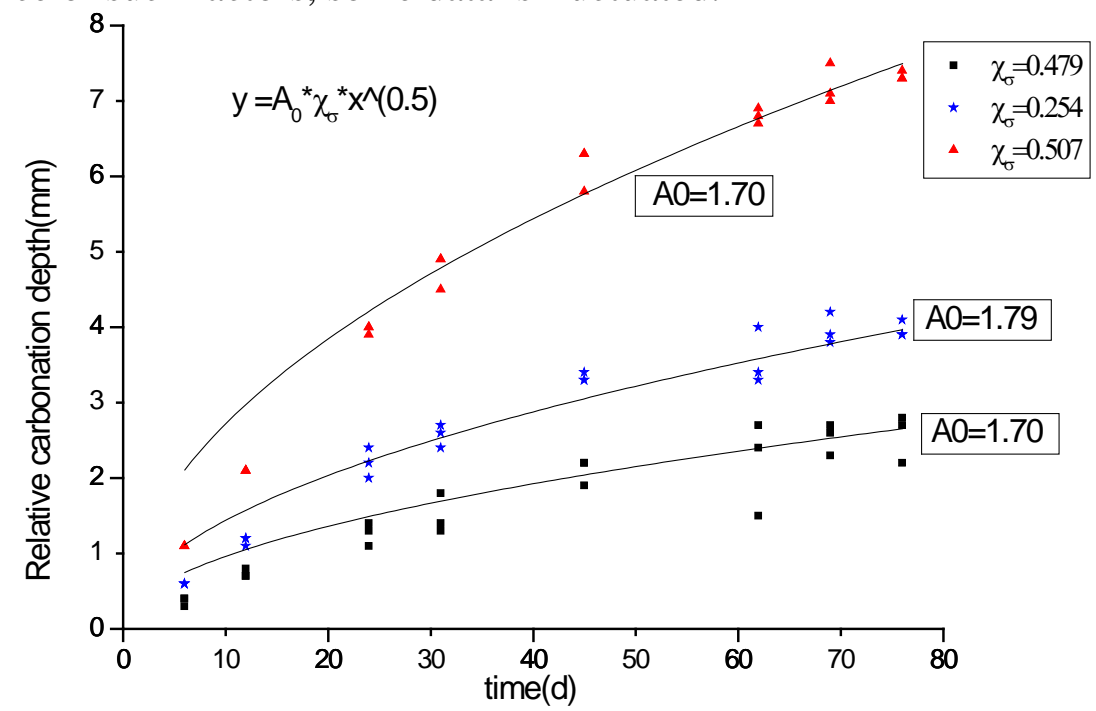

Fig 4.Relative Carbonation Depth function in tensile stress state

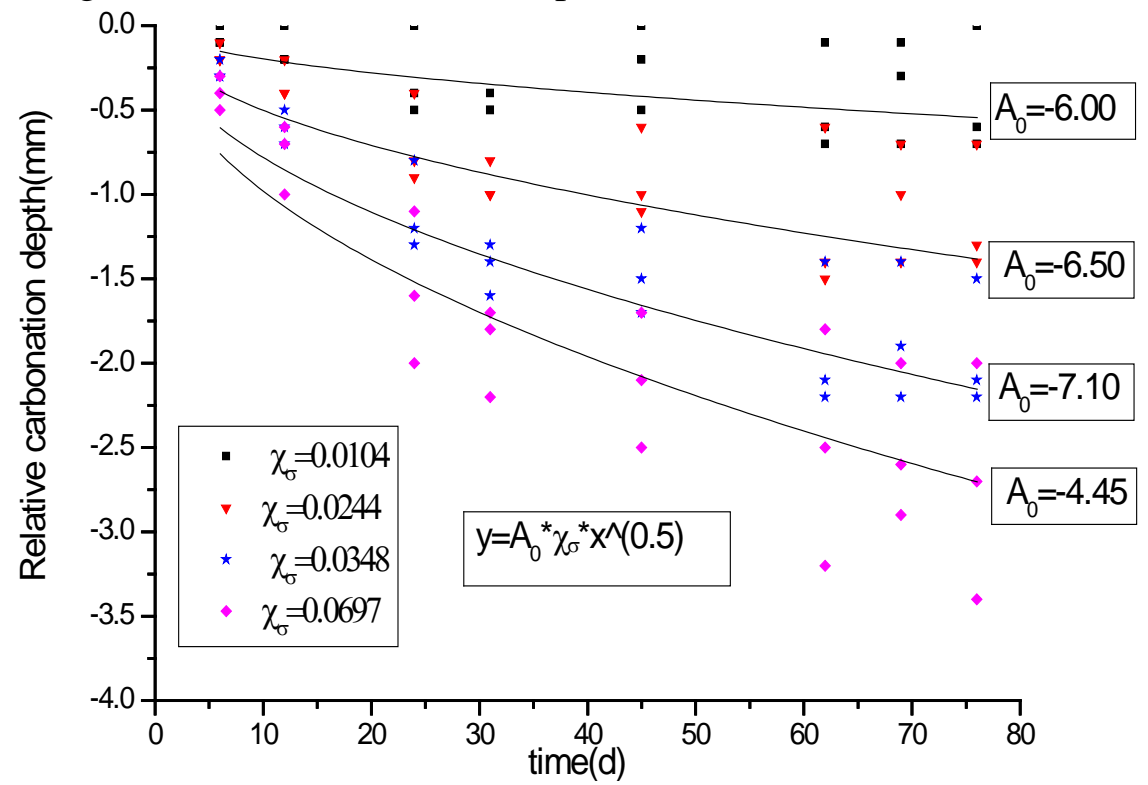

Fig 5. Relative Carbonation Depth function in compressive stress state

As shown in Fig 4 and Fig 5, the date from reference [6] was used to verify the formula (2).

In the tensile stress state, the coefficients of the formula (2) are shown in the Fig 4, while the coefficients in the compressive stress state are shown in the Fig 5. e

As shown in Fig4 and Fig5, the formula (2) can accurately predict the Relative Carbonation Depth in tensile stress state. When use it to predict the Relative Carbonation Depth in compressive stress state, the error is relative large. The main reasons for this Phenomenon are as follows:

1. The heterogeneity of concrete material itself, which leads to the Relative Carbonation Depth of a certain randomness.

2. In tensile stress state, the vales of the Relative Carbonation Depth are much larger, so the error is smaller. But in compressive state, the vales of the Relative Carbonation Depth are much smaller, so the error is relative large.

\section{Summary}

On the basis of summarizing the existing test data, put forward the method to calculate the carbonation depth of stress concrete components, and build a mathematical model. The main achieving as follows:

1. The carbonation depth of stress concrete components was divided into Standard Carbonation 
Depth and Relative Carbonation Depth; so the influence of stress on carbonation depth can be separated, and the data form different experiments can be used, the number of samples will be increased.

2. The Relative Carbonation Depth was put forward to consider the influence of stress, and the mathematical model of Relative Carbonation Depth was established and verified.

\section{Acknowledgement}

In this paper, the research was sponsored by the west of the ministry of transport construction projects of science and technology (Project No. 2013318223 040)

\section{References}

[1] Luo Xiaoyong, Zou Hongbo, SHI Qingliang, Experimental study on durability of concrete carbonation at different stress states, Journal of NaturalDisasters [J], 2012. 02 (21):194-198

[2] Shi Qingliang. The experiments research of concrete carbonation durability in stress state. Central South University, Master’s Degree thesis[D]. 2008:13-16.

[3] Tu Yongming, Lü Zhitao, Research on the experiment of prestressed concrete structures in carbonation environment and the prediction model of carbonation depth. Industrial construction[J]. 2006.01: 47-50

[4] Tu Yongming, Lü Zhitao. Experiment and research of presteressed concrete structure in carbonation corrosive environments[J].Journal of Southeast University(Natural Science Edition): 2003,33(5):573-576

[5] TIAN Hao, LI Guoping, LIU Jie, Wu Yongxian. Experimental Research on Carbonation of Forced Concrete Speciments[J]. Journal of Tongji University (Natural Science). 201002 (38) : 200-204

[6] Pan Hongke. Based on the carbonation of the durability and reliability of the underground structure[D]. Tongji University. Ph.D. Thesis. 2005:46-56.

[7] LIU Jie. Experments on the prestressed concrete structures durability[D]. Tongji University. Master's degree thesis. 2008:50-56

[8] LI Run-ji, DIAO Bo. Analysis of carbonation service life prediction model of reinforced concrete structures[J]. Concrete. 2009:232(02):4 7

[9] ZHANG Yu, JIANG LIxue, ZHANG Weiping, QU Wenjun. Durability of Concrete Structures[M]. Shanghai: Shanghai science and technology press. 2003,12:42-53.

[10]NIU Ditao. Durability and Life Forecast of Reinforced Concrete Structure[M]. Beijing: Science Press. 2003:158-163 